Bull. Korean Math. Soc. 47 (2010), No. 4, pp. 859-881

DOI 10.4134/BKMS.2010.47.4.859

\title{
HELICOIDAL SURFACES AND THEIR GAUSS MAP IN MINKOWSKI 3-SPACE
}

\author{
Miekyung Choi, Young Ho Kim, Huili Liu, and Dae Won Yoon
}

\begin{abstract}
The helicoidal surface is a generalization of rotation surface in a Minkowski space. We study helicoidal surfaces in a Minkowski 3-space in terms of their Gauss map and provide some examples of new classes of helicoidal surfaces with constant mean curvature in a Minkowski 3-space.
\end{abstract}

\section{Introduction}

As is well known that the helicoidal surface is a kind of generalization of some ruled surfaces and rotation surfaces in a Euclidean space or a Minkowski space. Quite a few works have been recently done with the helicoidal surfaces in Minkowski space with prescribed mean or Gaussian curvature $([3,10])$.

The notion of finite type immersion of submanifolds of a Euclidean space or a pseudo-Euclidean space has been widely used in classifying and characterizing well known Riemannian or pseudo-Riemannian submanifolds $([5,6])$. In some sense, it is a generalization of solving eigenvalue problems formed with the Laplace operator in the set of submanifolds in a Euclidean or a pseudoEuclidean space. It gives a nice relationship between some algebraic properties and geometric properties.

On the other hand, the Gauss map is a very useful tool to look into submanifolds of a Euclidean space or a pseudo-Euclidean space $([1,2,8,13])$. Thus, it is interesting to examine the behavior of the Gauss map of given submanifolds in a Euclidean space or a pseudo-Euclidean space based on the Laplacian. In particular, the Gauss map $G$ of some minimal (or maximal) surfaces including the catenoid in Euclidean 3-space and the Enneper's surface of the second kind in a Minkowski 3-space satisfies a unique partial differential equation similar to an eigenvalue problem that is not an actual eigenvalue problem.

Received March 10, 2009; Revised May 11, 2009.

2000 Mathematics Subject Classification. 53B25, 53C40.

Key words and phrases. helicoidal surfaces, Enneper's surface, Minkowski space, pointwise 1-type Gauss map.

The second author supported by KOSEF R01-2007-000-20014-0 (2007).

The third author supported by NSFC. 
Three of the present authors defined and used the notion of pointwise 1-type Gauss map to study certain surfaces in a Euclidean space or a Minkowski space $([7,9,12,13])$.

The Gauss map $G$ on a submanifold $M$ of a pseudo-Euclidean space $E_{s}^{m}$ of index $\mathrm{s}$ is said to be of pointwise 1-type if

$$
\Delta G=F(G+C)
$$

for a nonzero smooth function $F$ on $M$ and a constant vector $C$, where $\Delta$ denotes the Laplace operator defined on $M$. Especially, it is called proper if the function $F$ defined by (1.1) is non-constant. The non-proper pointwise 1-type Gauss map is just of 1-type in the usual sense $([5,8])$. A submanifold with pointwise 1-type Gauss map is said to be of the first kind if the vector $C$ in (1.1) is the zero vector. Otherwise, the pointwise 1-type Gauss map is said to be of the second kind $([7,9,12])$.

In this article we study the helicoidal surfaces with pointwise 1-type Gauss map in a Minkowski 3-space. We also provide some new examples of helicoidal surfaces in a Minkowski 3-space by solving some ordinary differential equations related to mean curvature.

\section{Preliminaries}

Let $\mathbb{E}_{1}^{3}$ be a Minkowski 3-space with the Lorentz metric

$$
\langle\cdot, \cdot\rangle=-d x_{0}^{2}+d x_{1}^{2}+d x_{2}^{2},
$$

where $\left(x_{0}, x_{1}, x_{2}\right)$ is a system of the canonical coordinates in $\mathbb{R}^{3}$. Let $M$ be a connected 2-dimensional surface in $\mathbb{E}_{1}^{3}$ and $x: M \rightarrow \mathbb{E}_{1}^{3}$ a smooth nondegenerate isometric immersion. A surface $M$ is said to be spacelike (resp. timelike) if the induced metric on $M$ is positive definite (resp. indefinite). Assuming that $M$ is orientable, we can always choose a unit normal vector field $G$ globally defined on $M$. On the other hand, the unit normal vector field $G$ can be regarded as a map $G: M \rightarrow \mathbb{H}_{+}^{2}$ if $M$ is spacelike, and as a map $G: M \rightarrow \mathbb{S}_{1}^{2}$ if $M$ is timelike. Here, $\mathbb{H}_{+}^{2}=\left\{x \in \mathbb{E}_{1}^{3} \mid\langle x, x\rangle=-1, x_{2}>0\right\}$ is the hyperbolic space and $\mathbb{S}_{1}^{2}=\left\{x \in \mathbb{E}_{1}^{3} \mid\langle x, x\rangle=1\right\}$ is the de Sitter space. The map $G$ is also called the Gauss map of the surface $M$. For the matrix $\tilde{g}=\left(\tilde{g}_{i j}\right)$ consisting of the components of the induced metric on $M$, we denote by $\tilde{g}^{-1}=\left(\tilde{g}^{i j}\right)$ (resp. $\mathcal{G}$ ) the inverse matrix (resp. the determinant) of the matrix $\left(\tilde{g}_{i j}\right)$. The Laplacian $\Delta$ on $M$ is, in turn, given by

$$
\Delta=-\frac{1}{\sqrt{|\mathcal{G}|}} \sum_{i, j} \frac{\partial}{\partial x^{i}}\left(\sqrt{|\mathcal{G}|} \tilde{g}^{i j} \frac{\partial}{\partial x^{j}}\right) .
$$

Let $e$ be a nonzero vector in $\mathbb{E}_{1}^{3}$ and $\mathbf{S}(e)$ be the set of screw motions fixing $e$ in $\mathbb{E}_{1}^{3}$. In particular, if $e$ is non-null, the screw motions fixing $e$ belong to $\mathbf{O}(e)$, the set of orthogonal transformations with positive determinant. Then 
a helicoidal motion around the axis in the $e$-direction can be defined by

$$
g_{t}(x)=A(t) x^{T}+(h t) e^{T}, \quad x=\left(x_{0}, x_{1}, x_{2}\right) \in \mathbb{E}_{1}^{3}, t \in \mathbb{R}, A \in \mathbf{S}(e),
$$

where $h$ is a constant.

Let $\gamma: I=(a, b) \subset \mathbb{R} \rightarrow \Pi$ be a plane curve in $\mathbb{E}_{1}^{3}$ and $l$ a straight line in $\Pi$ which does not intersect the curve $\gamma$. A helicoidal surface $M$ with the axis $l$ and pitch $h$ in $\mathbb{E}_{1}^{3}$ is a nondegenerate surface which is invariant under the action of the helicoidal motion $g_{t}$. Depending on the axis being spacelike, timelike or null, there are three types of screw motions. If the axis $l$ is spacelike (resp. timelike), then $l$ is transformed to the $x_{1}$-axis or $x_{2}$-axis (resp. $x_{0}$-axis) by the Lorentz transformation. Therefore, we may consider $x_{2}$-axis (resp. $x_{0}$-axis) as the axis if $l$ is spacelike (resp. timelike). If the axis $l$ is null, then we may assume that the axis is the line spanned by the vector $(1,1,0)$.

We now consider the helicoidal surfaces in $\mathbb{E}_{1}^{3}$ with spacelike, timelike or null axis respectively.

Case 1. The axis $l$ is spacelike.

Without loss of generality we may assume that the profile curve $\gamma$ lies in the $x_{1} x_{2}$-plane or $x_{0} x_{2}$-plane. Hence the curve $\gamma$ can be represented by

$$
\gamma(u)=(0, f(u), g(u)) \quad \text { or } \quad \gamma(u)=(f(u), 0, g(u))
$$

for smooth functions $f$ and $g$ on an open interval $I=(a, b)$. Therefore, the surface $M$ may be parameterized by

$$
x(u, v)=(f(u) \sinh v, f(u) \cosh v, g(u)+h v), f(u)>0, h \in \mathbb{R}
$$

or

$$
x(u, v)=(f(u) \cosh v, f(u) \sinh v, g(u)+h v), f(u)>0, h \in \mathbb{R} .
$$

Case 2. The axis $l$ is timelike.

In this case, we may assume that the curve $\gamma$ lies in the $x_{0} x_{1}$-plane. So the curve $\gamma$ is given by $\gamma(u)=(g(u), f(u), 0)$ for a positive function $f=f(u)$ on an open interval $I=(a, b)$. Hence the surface $M$ can be expressed by

$$
x(u, v)=(g(u)+h v, f(u) \cos v, f(u) \sin v), f(u)>0, h \in \mathbb{R} .
$$

Case 3. The axis $l$ is null.

In this case, we may assume that the curve $\gamma$ lies in the $x_{0} x_{1}$-plane of the form $\gamma(u)=(f(u), g(u), 0)$, where $f=f(u)$ is a positive function and $g=g(u)$ is a function satisfying $p(u)=f(u)-g(u) \neq 0$ for all $u \in I$. Under the cubic screw motions, its parametrization has the form

$$
x(u, v)=\left(f(u)+\frac{v^{2}}{2} p(u)+h v, g(u)+\frac{v^{2}}{2} p(u)+h v, p(u) v\right), h \in \mathbb{R} .
$$




\section{Examples of helicoidal surfaces with pointwise 1-type Gauss map}

In this section, we provide some examples of helicoidal surfaces with pointwise 1-type Gauss map in Minkowski 3-space and we give the names of them upon their invariance under the orthogonal transformations related to those of rotation surfaces in $\mathbb{E}_{1}^{3}$.

Example 3.1 (Right helicoid of type $I$ ). A right helicoid with spacelike axis in $\mathbb{E}_{1}^{3}$ is parameterized by

$$
x(u, v)=(u \sinh v, u \cosh v, a+h v), u>0, h \neq 0
$$

for some constant $a$. It could be spacelike or timelike depending on the region satisfying either $-u^{2}+h^{2}>0$ or $-u^{2}+h^{2}<0$. If it is spacelike, its Gauss map $G$ is given by

$$
G=\frac{1}{\sqrt{-u^{2}+h^{2}}}(-h \cosh v,-h \sinh v,-u) .
$$

Hence the Laplacian $\Delta G$ of the Gauss map $G$ satisfies

$$
\Delta G=-\frac{2 h^{2}}{\left(-u^{2}+h^{2}\right)^{2}} G,
$$

which has pointwise 1-type Gauss map of the first kind. In case of $M$ being timelike, we can have a similar result.

\section{Fig 1 : Right helicoid of type I}

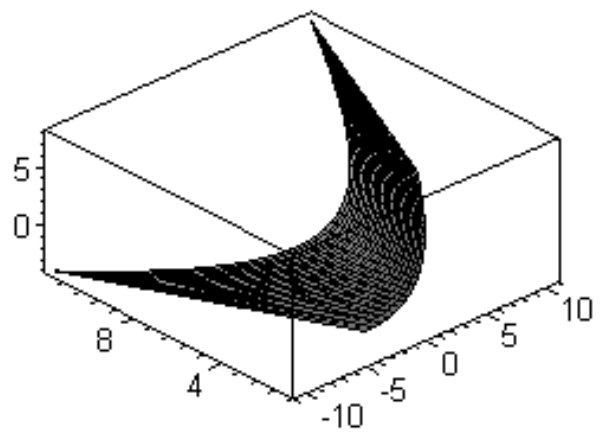

Example 3.2 (Right helicoid of type $I I$ ). A right helicoid $M$ with timelike axis in $\mathbb{E}_{1}^{3}$ is parameterized by

$$
x(u, v)=(a+h v, u \cos v, u \sin v), u>0, h \neq 0
$$

for some constant $a$. The surface $M$ is spacelike or timelike upon the sign of $h^{2}-u^{2}$. Assuming that $M$ is spacelike, the Gauss map $G$ is given by

$$
G=\frac{1}{\sqrt{u^{2}-h^{2}}}(-u, h \sin v,-h \cos v) .
$$


By a direct computation, we see that its Laplacian satisfies

$$
\Delta G=-\frac{2 h^{2}}{\left(u^{2}-h^{2}\right)^{2}} G
$$

and hence, the Gauss map is of pointwise 1-type of the first kind.

Fig 2: Right helicoid of type ॥

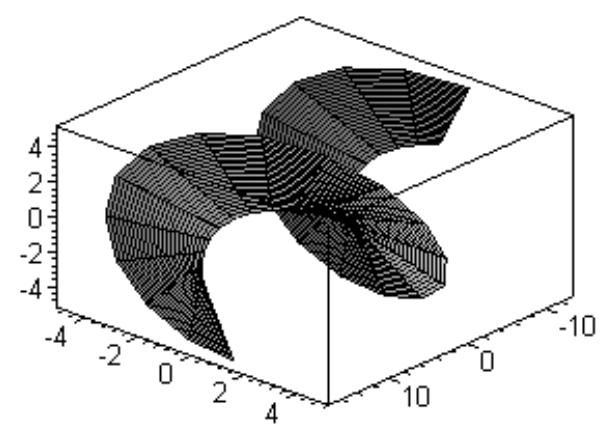

Example 3.3 (Helicoidal surface of elliptic type). Let $M$ be a surface with timelike axis in $\mathbb{E}_{1}^{3}$ parameterized by

$$
x(u, v)=( \pm u+a+h v, u \cos v, u \sin v), u>0, h \neq 0
$$

for some constant $a$. Then, the Gauss map $G$ is obtained by

$$
G=\frac{1}{|h|}(-u, \mp u \cos v+h \sin v, \mp u \sin v-h \cos v) \text {. }
$$

Therefore, its Laplacian satisfies

$$
\Delta G=\frac{2}{h^{2}} G
$$

and thus, the Gauss map $G$ of $M$ is of pointwise 1-type of the first kind. Indeed, it is non-proper. We call such a surface $M$ a helicoidal surface of elliptic type.

Fig 3: Helicoidal surface of elliptic type

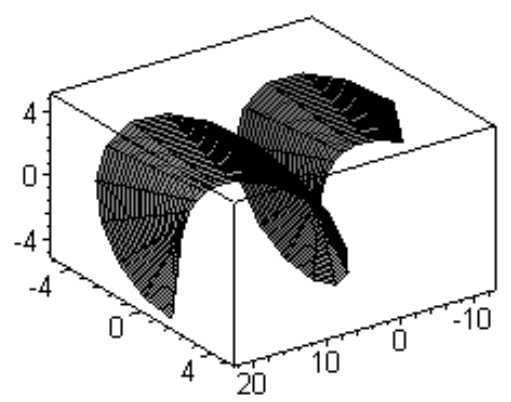


The following helicoidal surfaces are generated by a null axis.

Example 3.4 (Spacelike helicoidal surface of Enneper type). For a positive constant $a$ and a constant $b$, a helicoidal surface parameterized by

$x(u, v)=\left(a u^{3}-\frac{h^{2}}{4 u}+b-u-u v^{2}+h v, a u^{3}-\frac{h^{2}}{4 u}+b+u-u v^{2}+h v,-2 u v\right)$

has the Gauss map

$G=\frac{1}{2 \sqrt{3 a} u^{2}}\left(3 a u^{3}+\frac{h^{2}}{4 u}+u+u v^{2}-h v, 3 a u^{3}+\frac{h^{2}}{4 u}-u+u v^{2}-h v, 2 u v-h\right)$.

Then, the Gauss map satisfies

$$
\Delta G=-\frac{1}{6 a u^{4}} G .
$$

Thus, it has pointwise 1-type Gauss map of the first kind and it is proved to be minimal. We call such a surface a helicoidal surface of Enneper type.

Fig 4: Helicoidal surface of Enneper type

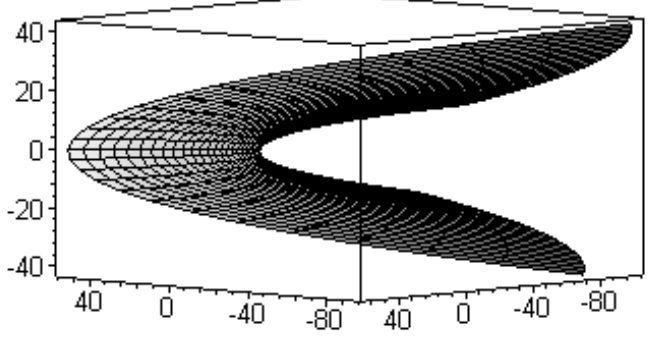

Example 3.5 (Helicoidal surface of hyperbolic type or de Sitter type). Let $M$ be a helicoidal surface parameterized by

$x(u, v)=\left(-\frac{a}{u}-\frac{h^{2}}{4 u}+b-u-u v^{2}+h v,-\frac{a}{u}-\frac{h^{2}}{4 u}+b+u-u v^{2}+h v,-2 u v\right)$ for some positive constant $a$ and some constant $b$. Then, the Gauss map $G$ is given by

$$
G=\frac{1}{2 \sqrt{a}}\left(\frac{a}{u}+\frac{h^{2}}{4 u}+u+u v^{2}-v h, \frac{a}{u}+\frac{h^{2}}{4 u}-u+u v^{2}-v h, 2 u v-h\right) .
$$

Hence, the Laplacian $\Delta G$ is given by

$$
\Delta G=-\frac{1}{2 a} G
$$


Thus, it has non-proper pointwise 1-type Gauss map of the first kind. In fact, it is easily proved that $M$ has nonzero constant mean curvature. Such a surface $M$ is called a helicoidal surface of hyperbolic type. The surface $M$ is called a helicoidal surface of de Sitter type if the constant $a$ is negative.

Fig 5: Helicoidal surface of de Sitter type

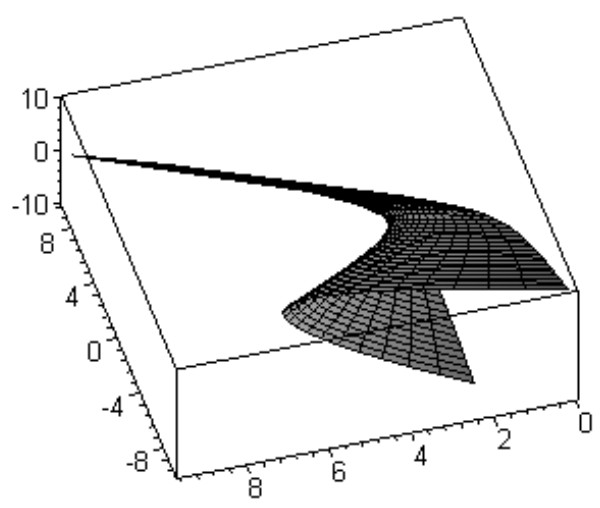

Example 3.6 (Helicoidal surface of parabolic type $I^{+}$). Consider a spacelike helicoidal surface parameterized by

$$
x(u, v)=\left(k(u)-u-u v^{2}+h v, k(u)+u-u v^{2}+h v,-2 u v\right), h \neq 0,
$$

where $k(u)=-\frac{1}{4}\left(\frac{h^{2}}{u}+\frac{u}{2\left(u^{2}+1\right)}-\frac{\tan ^{-1} u}{2}\right)$. Then, the Gauss map is given by $G=\frac{u^{2}+1}{u^{2}}\left(\frac{h^{2}}{4 u}+\frac{u^{3}}{4\left(u^{2}+1\right)^{2}}+u+u v^{2}-v h, \frac{h^{2}}{4 u}+\frac{u^{3}}{4\left(u^{2}+1\right)^{2}}-u+u v^{2}-v h, 2 u v-h\right)$. Moreover, its Laplacian satisfies

$$
\Delta G=\frac{-2\left(u^{4}+1\right)}{u^{4}} G .
$$

Thus, $G$ is of pointwise 1-type of the first kind. The helicoidal surface parameterized as above is called a helicoidal surface of parabolic type $I^{+}$.

Example 3.7 (Helicoidal surface of parabolic type $I I^{+}$). Let a helicoidal surface be parameterized by

$$
x(u, v)=\left(k(u)-u-u v^{2}+h v, k(u)+u-u v^{2}+h v,-2 u v\right), h \neq 0,
$$

where $k(u)=-\frac{1}{4}\left(\frac{h^{2}}{u}-\frac{u}{2\left(u^{2}+1\right)}+\frac{\tan ^{-1} u}{2}\right)$. Then, it is timelike and the Gauss map $G$ can be expressed by

$G=\frac{u^{2}+1}{u^{2}}\left(\frac{h^{2}}{4 u}-\frac{u^{3}}{4\left(u^{2}+1\right)^{2}}+u+u v^{2}-v h, \frac{h^{2}}{4 u}-\frac{u^{3}}{4\left(u^{2}+1\right)^{2}}-u+u v^{2}-v h, 2 u v-h\right)$. 
Fig 6: Helicoidal surface of parabolic type $\left.\right|^{n}+$

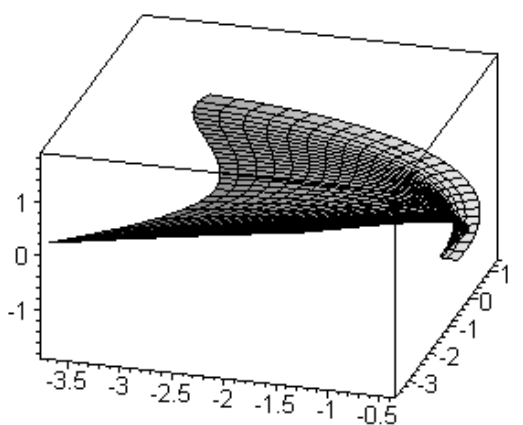

A direct computation yields

$$
\Delta G=\frac{2\left(u^{4}+1\right)}{u^{4}} G .
$$

So, it has pointwise 1-type Gauss map of the first kind. The helicoidal surface above is called a helicoidal surface of parabolic type $\mathrm{II}^{+}$.

Fig 7 : Helicoidal surface of parabolic type $\|^{\mu_{+}}$

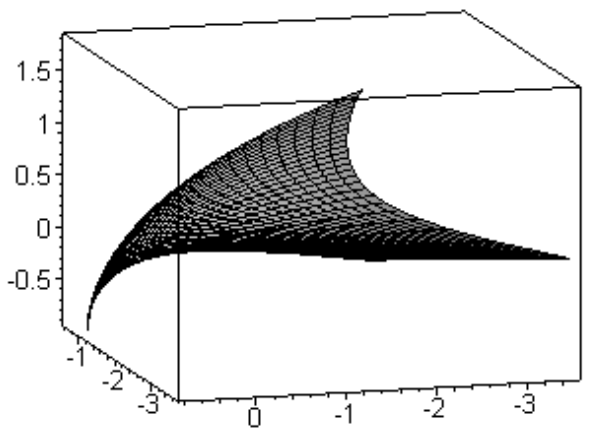

4. Fundamental lemma

We prove the following lemma for later use.

Lemma 4.1. Let $M$ be a helicoidal surface in a Minkowski 3-space. If $M$ has pointwise 1-type Gauss map, then the function $F$ in (1.1) depends only on $u$ and the vector $C$ is parallel to the axis in $\mathbb{E}_{1}^{3}$. 
Proof. We separate three cases of proof according to the character of the axis.

Case 1. Suppose that $M$ is a helicoidal surface in $\mathbb{E}_{1}^{3}$ with spacelike axis parameterized by (2.1) for some smooth functions $f$ and $g$.

First, if $f$ is constant, then the parametrization of $M$ can be written as

$$
x(u, v)=(a \sinh v, a \cosh v, g(u)+h v), h \in \mathbb{R}
$$

for a nonzero constant $a$. From a direct computation, we see that the Laplacian $\Delta G$ of the Gauss map $G$ satisfies $\Delta G=\frac{1}{a^{2}} G$. Therefore $M$ has non-proper pointwise 1-type Gauss map of the first kind, that is, the constant vector $C$ is zero vector. In this case, $M$ is part of a hyperbolic cylinder.

From now on, we assume that $f$ is not a constant function. Then, we may put $f(u)=u$. Thus, $M$ is parameterized by

$$
x(u, v)=(u \sinh v, u \cosh v, g(u)+h v), u>0, h \in \mathbb{R} .
$$

We now suppose that $M$ is timelike. By a direct computation, the Gauss map $G$ and its Laplacian $\Delta G$ are obtained as follows:

$$
G=\frac{1}{\sqrt{u^{2}+u^{2} g^{\prime 2}-h^{2}}}\left(-h \cosh v+u g^{\prime} \sinh v,-h \sinh v+u g^{\prime} \cosh v,-u\right)
$$

$$
\Delta G=-\frac{1}{\left(u^{2}+u^{2} g^{\prime 2}-h^{2}\right)^{\frac{7}{2}}}\left(A_{1} \cosh v+B_{1} \sinh v, A_{1} \sinh v+B_{1} \cosh v, D_{1}\right),
$$

where we have put

$$
\begin{aligned}
A_{1}= & A_{1}(u) \\
= & h\left\{2 h^{4}+4 h^{4} g^{\prime 2}+7 h^{4} g^{\prime} g^{\prime \prime} u+\left(-2 h^{2}-2 h^{2} g^{2}+h^{4} g^{\prime \prime 2}+h^{4} g^{\prime} g^{\prime \prime \prime}\right) u^{2}\right. \\
& +\left(-8 h^{2} g^{\prime} g^{\prime \prime}+h^{2} g^{3} g^{\prime \prime}\right) u^{3} \\
& +\left(3 h^{2} g^{\prime 2} g^{\prime \prime 2}-h^{2} g^{\prime 3} g^{\prime \prime \prime}-2 h^{2} g^{\prime \prime 2}-2 h^{2} g^{\prime} g^{\prime \prime \prime}\right) u^{4} \\
& \left.+\left(g^{\prime} g^{\prime \prime}+g^{\prime 3} g^{\prime \prime}\right) u^{5}+\left(g^{\prime \prime 2}-3 g^{\prime 2} g^{\prime \prime 2}+g^{\prime} g^{\prime \prime \prime}+g^{\prime 3} g^{\prime \prime \prime}\right) u^{6}\right\},
\end{aligned}
$$

$$
\begin{aligned}
B_{1}= & B_{1}(u) \\
= & -3 h^{6} g^{\prime \prime}+\left(-6 h^{4} g^{\prime}-8 h^{4} g^{3}-h^{6} g^{\prime \prime \prime}\right) u+\left(7 h^{4} g^{\prime \prime}-7 h^{4} g^{2} g^{\prime \prime}\right) u^{2} \\
& +\left(7 h^{2} g^{\prime}+12 h^{2} g^{3}+5 h^{2} g^{\prime 5}-4 h^{4} g^{\prime} g^{\prime \prime 2}+3 h^{4} g^{\prime \prime \prime}+h^{4} g^{\prime 2} g^{\prime \prime \prime}\right) u^{3} \\
& +\left(-5 h^{2} g^{\prime \prime}+6 h^{2} g^{\prime 2} g^{\prime \prime}+2 h^{2} g^{\prime 4} g^{\prime \prime}\right) u^{4}+\left(-g^{\prime}\left(1+g^{2}\right)^{3}+8 h^{2} g^{\prime} g^{\prime \prime 2}\right. \\
& \left.-3 h^{2} g^{\prime \prime \prime}-2 h^{2} g^{\prime 2} g^{\prime \prime \prime}\right) u^{5}+\left(g^{\prime \prime}+g^{\prime 2} g^{\prime \prime}\right) u^{6}+\left(g^{\prime 2} g^{\prime \prime \prime}-4 g^{\prime} g^{\prime \prime 2}+g^{\prime \prime \prime}\right) u^{7}
\end{aligned}
$$


and

(4.7)

$$
\begin{aligned}
D_{1}= & D_{1}(u) \\
= & u\left\{\left(2 h^{4}+4 h^{4} g^{\prime 2}\right)+7 h^{4} g^{\prime} g^{\prime \prime} u+\left(-2 h^{2}-2 h^{2} g^{2}+h^{4} g^{\prime \prime 2}+h^{4} g^{\prime} g^{\prime \prime \prime}\right) u^{2}\right. \\
& +\left(-8 h^{2} g^{\prime} g^{\prime \prime}+h^{2} g^{\prime 3} g^{\prime \prime}\right) u^{3}+\left(3 h^{2} g^{\prime 2} g^{\prime \prime 2}-h^{2} g^{\prime 3} g^{\prime \prime \prime}-2 h^{2} g^{\prime \prime 2}\right. \\
& \left.\left.-2 h^{2} g^{\prime} g^{\prime \prime \prime}\right) u^{4}+\left(g^{\prime} g^{\prime \prime}+g^{\prime 3} g^{\prime \prime}\right) u^{5}+\left(g^{\prime \prime 2}-3 g^{\prime 2} g^{\prime \prime 2}+g^{\prime} g^{\prime \prime \prime}+g^{\prime 3} g^{\prime \prime \prime}\right) u^{6}\right\} .
\end{aligned}
$$

Suppose that the Gauss map $G$ of $M$ is of pointwise 1-type, that is, (1.1) holds on $M$. With the help of (4.3) and (4.4), it implies that the function $F$ is independent of the parameter $v$, that is, $F$ depends only on $u$. Moreover, the first two components of constant vector $C$ are zero, that is, $C=(0,0, c)$ for some constant $c$. In other words, the constant vector $C$ is parallel to the axis.

By a similar argument as above, we have the same results in case of spacelike helicoidal surfaces with spacelike axis.

Case 2. Suppose that $M$ is a helicoidal surface with timelike axis parameterized by (2.3) for some smooth functions $f$ and $g$.

If $f$ is constant, then $M$ is part of an ordinary circular cylinder parameterized by

$$
x(u, v)=(g(u)+h v, a \cos v, a \sin v), h \in \mathbb{R}
$$

for a nonzero constant $a$. Then, its Gauss map $G$ satisfies $\Delta G=\frac{1}{a^{2}} G$ and thus it is of non-proper pointwise 1-type of the first kind.

We now consider that $f$ is not constant. Putting $f(u)=u$, the parametrization of $M$ can be written as

$$
x(u, v)=(g(u)+h v, u \cos v, u \sin v), u>0, h \in \mathbb{R} .
$$

If $M$ is timelike, we derive the Gauss map $G$ and its Laplacian $\Delta G$ as follows:

$$
G=\frac{1}{\sqrt{-u^{2}+u^{2} g^{\prime 2}+h^{2}}}\left(-u,-u g^{\prime} \cos v+h \sin v,-u g^{\prime} \sin v-h \cos v\right)
$$

and

$$
\Delta G=-\frac{1}{\left(-u^{2}+u^{2} g^{\prime 2}+h^{2}\right)^{\frac{7}{2}}}\left(D_{2}, A_{2} \sin v+B_{2} \cos v,-A_{2} \cos v+B_{2} \sin v\right),
$$

where $A_{2}, B_{2}$ and $D_{2}$ are some functions of $u$ similarly defined by (4.5), (4.6) and (4.7).

If $M$ satisfies (1.1), we see that the function $F$ depends only on $u$ by using (4.10) and (4.11) similarly as we developed in Case 1 and the vector $C$ is parallel to the axis.

The same conclusion can be made in case of spacelike helicoidal surfaces with timelike axis. 
Case 3. Suppose that $M$ is a helicoidal surface with null axis parameterized by

$$
x(u, v)=\left(f(u)+\frac{v^{2}}{2} p(u)+h v, g(u)+\frac{v^{2}}{2} p(u)+h v, p(u) v\right), h \in \mathbb{R},
$$

where $p(u)=f(u)-g(u) \neq 0$.

Since the induced metric on $M$ is nondegenerate, $(f(u)-g(u))^{2}\left(f^{\prime 2}(u)-\right.$ $\left.g^{\prime 2}(u)\right)+h^{2}\left(f^{\prime}(u)-g^{\prime}(u)\right)^{2}$ never vanishes and so $f^{\prime}(u)-g^{\prime}(u) \neq 0$ everywhere. Thus, we may change the variable in such a way that $p(u)=f(u)-g(u)=-2 u$.

Let $k(u)=f(u)+u$. Then, the functions $f$ and $g$ in the profile curve $\gamma$ look like

$$
f(u)=k(u)-u \quad \text { and } \quad g(u)=k(u)+u .
$$

Thus, the parametrization of $M$ becomes

$$
x(u, v)=\left(k(u)-u-u v^{2}+h v, k(u)+u-u v^{2}+h v,-2 u v\right) .
$$

Since $M$ is nondegenerate, $4 u^{2} k^{\prime}(u)-h^{2} \neq 0$ everywhere it is defined. So, we get the Gauss map $G$ and the Laplacian $\Delta G$ of $G$ as follows:

$G=\frac{1}{\sqrt{\left|4 u^{2} k^{\prime}(u)-h^{2}\right|}}\left(u k^{\prime}(u)+u+u v^{2}-v h, u k^{\prime}(u)-u+u v^{2}-v h, 2 u v-h\right)$

and

$$
\Delta G=-\frac{1}{\left|4 u^{2} k^{\prime}(u)-h^{2}\right|^{\frac{7}{2}}}(2 u X+Y,-2 u X+Y, 2(2 u v-h) X),
$$

where we have put

$X=X(u)=h^{4}+4 h^{2} k^{\prime} u^{2}+9 h^{2} k^{\prime \prime} u^{3}+h^{2} k^{\prime \prime \prime} u^{4}-4 k^{\prime} k^{\prime \prime} u^{5}+8 k^{\prime \prime 2} u^{6}-4 k^{\prime} k^{\prime \prime \prime} u^{6}$ and

$$
\begin{aligned}
(4.16) & Y(u, v) \\
= & 10 h^{4} k^{\prime} u+7 h^{4} k^{\prime \prime} u^{2}-32 h^{2} k^{\prime 2} u^{3}+h^{4} k^{\prime \prime \prime} u^{3}-14 h^{2} k^{\prime} k^{\prime \prime} u^{4}+32 k^{3} u^{5} \\
& +6 h^{2} k^{\prime \prime 2} u^{5}-6 h^{2} k^{\prime} k^{\prime \prime \prime} u^{5}+8 k^{\prime 2} k^{\prime \prime} u^{6}-8 k^{\prime} k^{\prime \prime 2} u^{7}+8 k^{\prime 2} k^{\prime \prime \prime} u^{7}-2 h^{5} v \\
& -8 h^{3} k^{\prime} u^{2} v-18 h^{3} k^{\prime \prime} u^{3} v-2 h^{3} k^{\prime \prime \prime} u^{4} v+8 h k^{\prime} k^{\prime \prime} u^{5} v-16 h k^{\prime \prime} u^{6} v \\
& +8 h k^{\prime} k^{\prime \prime \prime} u^{6} v+2 h^{4} u v^{2}+8 h^{2} k^{\prime} u^{3} v^{2}+18 h^{2} k^{\prime \prime} u^{4} v^{2}+2 h^{2} k^{\prime \prime \prime} u^{5} v^{2} \\
& -8 k^{\prime} k^{\prime \prime} u^{6} v^{2}+16 k^{\prime \prime} u^{7} v^{2}-8 k^{\prime} k^{\prime \prime \prime} u^{7} v^{2} .
\end{aligned}
$$

We now suppose that $M$ is spacelike, that is, $4 u^{2} k^{\prime}(u)-h^{2}>0$ and the Gauss map $G$ is of pointwise 1-type.

Let $(\Delta G)_{i}$ be the $i$-th component of $\Delta G$ for $i=1,2,3$. Then, we have

$$
(\Delta G)_{1}=F(u, v)\left(\frac{u k^{\prime}+u+u v^{2}-v h}{\sqrt{4 u^{2} k^{\prime}-h^{2}}}+c_{1}\right),
$$




$$
\begin{gathered}
(\Delta G)_{2}=F(u, v)\left(\frac{u k^{\prime}-u+u v^{2}-v h}{\sqrt{4 u^{2} k^{\prime}-h^{2}}}+c_{2}\right), \\
(\Delta G)_{3}=F(u, v)\left(\frac{2 u v-h}{\sqrt{4 u^{2} k^{\prime}-h^{2}}}+c_{3}\right),
\end{gathered}
$$

where $C=\left(c_{1}, c_{2}, c_{3}\right)$. Subtracting (4.18) from (4.17), we get

$$
-\frac{4 u X(u)}{\left(4 u^{2} k^{\prime}-h^{2}\right)^{\frac{7}{2}}}=F(u, v)\left(\frac{2 u}{\sqrt{4 u^{2} k^{\prime}-h^{2}}}+c_{1}-c_{2}\right) .
$$

Hence, we see that the function $F$ depends only on $u$, that is, $F(u, v)=F(u)$. Differentiating the equation (4.19) with respect to the parameter $v$, we obtain

$$
-\frac{4 u X(u)}{\left(4 u^{2} k^{\prime}-h^{2}\right)^{\frac{7}{2}}}=F(u) \frac{2 u}{\sqrt{4 u^{2} k^{\prime}-h^{2}}} .
$$

With the help of (4.20) and (4.21), we have $c_{1}=c_{2}$ and moreover,

$$
F(u)=-\frac{2 X(u)}{\left(4 u^{2} k^{\prime}-h^{2}\right)^{3}} .
$$

Putting (4.22) in (4.19), we obtain $c_{3}=0$. This means that the constant vector $C$ is parallel to the axis, that is, $C=(c, c, 0)$ for some constant c.

A similar conclusion is achieved in case that $M$ is timelike, that is, $4 u^{2} k^{\prime}(u)-$ $h^{2}<0$. Thus, the proof of lemma is completed.

\section{Helicoidal surfaces with spacelike axis in $\mathbb{E}_{1}^{3}$}

In this section, we study the helicoidal surface with spacelike axis in $\mathbb{E}_{1}^{3}$.

Let $M$ be a helicoidal surface with spacelike axis parameterized by (4.2) for some smooth function $g$, which has pointwise 1-type Gauss map, that is, $\Delta G=F(G+C)$ for a nonzero smooth function $F$ and a constant vector $C$. By Lemma 4.1, the Laplacian of the Gauss map satisfies $\Delta G=F(u)(G+(0,0, c))$ for some constant $c$. First, we assume that $M$ is timelike, i.e., $u^{2} g^{\prime 2}+u^{2}-h^{2}>$ 0 . Then, from (4.3) and (4.4), we have $A_{1}, B_{1}$ and $D_{1}$ as follows

$$
\begin{aligned}
& A_{1}=h F\left(u^{2} g^{\prime 2}+u^{2}-h^{2}\right)^{3}, \\
& B_{1}=-F u g^{\prime}\left(u^{2} g^{\prime 2}+u^{2}-h^{2}\right)^{3}, \\
& D_{1}=F\left(u^{2} g^{\prime 2}+u^{2}-h^{2}\right)^{3}\left(u-c \sqrt{u^{2} g^{\prime 2}+u^{2}-h^{2}}\right) .
\end{aligned}
$$

We now assume that $M$ is a genuine helicoidal surface, that is, $h \neq 0$. From the above equations, we have

$$
B_{1}+u g^{\prime} \frac{A_{1}}{h}=0 \quad \text { and } \quad D_{1}=\frac{A_{1}}{h}\left(u-c \sqrt{u^{2} g^{\prime 2}+u^{2}-h^{2}}\right) .
$$

With the help of (4.5), (4.7) and (5.1), we get $c F\left(u^{2} g^{\prime 2}+u^{2}-h^{2}\right)^{\frac{7}{2}}=0$. Since $F$ is a nonzero function and $u^{2} g^{\prime 2}+u^{2}-h^{2} \neq 0$ everywhere, we obtain $c=0$. 
Therefore, the Gauss map $G$ is of pointwise 1-type of the first kind. Similarly, we can derive the same conclusion for the spacelike case. Thus, we have:

Theorem 5.1. Let $M$ be a genuine helicoidal surface with spacelike axis in a Minkowski 3-space $\mathbb{E}_{1}^{3}$. If the Gauss map $G$ of $M$ is of pointwise 1-type, then it is of the first kind, that is, the Gauss map satisfies the equation $\Delta G=F G$ for some nonzero smooth function $F$.

By Lemma 5.1 in [13], if $M$ is a surface in a Minkowski 3-space $\mathbb{E}_{1}^{3}$ with pointwise 1-type Gauss map of the first kind, then its mean curvature is constant. Hence, we have:

Corollary 5.2. Let $M$ be a genuine helicoidal surface with spacelike axis in a Minkowski 3-space $\mathbb{E}_{1}^{3}$. Then $M$ has pointwise 1-type Gauss map if and only if $M$ has constant mean curvature.

We now prove:

Theorem 5.3. A genuine helicoidal surface $M$ with spacelike axis in a Minkowski 3-space $\mathbb{E}_{1}^{3}$ has pointwise 1-type Gauss map if and only if it is an open part of either a hyperbolic cylinder parameterized by (4.1) or the surface parameterized by

$$
x(u, v)=(u \sinh v, u \cosh v, g(u)+h v), u>0, h \neq 0,
$$

where

$$
g(u)= \begin{cases} \pm \int\left(\alpha u+\frac{a}{u}\right) \sqrt{\frac{u^{2}-h^{2}}{u^{2}-\left(\alpha u^{2}+a\right)^{2}}} d u \quad \text { if } M \text { is timelike } \\ \pm \int\left(\alpha u+\frac{a}{u}\right) \sqrt{\frac{-u^{2}+h^{2}}{u^{2}+\left(\alpha u^{2}+a\right)^{2}}} d u \quad \text { if } M \text { is spacelike }\end{cases}
$$

for some constant $a$ and constant mean curvature $\alpha$.

Proof. Suppose that $M$ is a genuine helicoidal surface with spacelike axis in $\mathbb{E}_{1}^{3}$ with pointwise 1-type Gauss map. Then, by Theorem 5.1 and Corollary 5.2, it is of the first kind and mean curvature is constant.

If the function $f$ in the parametrization (2.1) is constant, then $M$ is part of a hyperbolic cylinder as is shown in Lemma 4.1.

We now suppose that $f$ is not constant. By (4.2), the parametrization of $M$ is given by

$$
x(u, v)=(u \sinh v, u \cosh v, g(u)+h v), u>0, h \in \mathbb{R} .
$$

First, consider the case that $M$ is timelike, that is, $-u^{2}+h^{2}-u^{2} g^{\prime 2}<0$. Then, $M$ has constant mean curvature $\alpha$ if and only if $g=g(u)$ is a solution of the following differential equation

$$
h^{2} u g^{\prime \prime}-u^{3} g^{\prime \prime}+2 h^{2} g^{\prime}-u^{2} g^{\prime}-u^{2} g^{\prime 3}=2 \alpha\left(u^{2}-h^{2}+u^{2} g^{\prime 2}\right)^{\frac{3}{2}} .
$$

If $u^{2}-h^{2}>0$, we put $u^{2}-h^{2}=w^{2}$. By the change of variables $\frac{u}{w} g^{\prime}=\tan y$, the equation (5.2) becomes

$$
-w^{3} \sec ^{2} y y^{\prime}-\frac{w^{3}}{u} \tan y \sec ^{2} y=2 \alpha w^{3} \sec ^{3} y .
$$


It follows

$$
-u \cos y y^{\prime}-\sin y=2 \alpha u,
$$

which yields $\sin y=-\alpha u-\frac{a}{u}$ for some constant $a$. Thus, the function $g(u)$ is obtained by

$$
g(u)= \pm \int\left(\alpha u+\frac{a}{u}\right) \sqrt{\frac{u^{2}-h^{2}}{u^{2}-\left(\alpha u^{2}+a\right)^{2}}} d u .
$$

Similarly, if $u^{2}-h^{2}<0$, the change of variables $u^{2}-h^{2}=-w^{2}$ and $\frac{u}{w} g^{\prime}=\sec y$ enables (5.2) to be

$$
w^{3} \sec y \tan y y^{\prime}-\frac{w^{3}}{u} \sec y \tan ^{2} y=2 \alpha w^{3} \tan ^{3} y .
$$

From this, the same result as above is derived. Consequently, if $M$ is a timelike surface with constant mean curvature $\alpha$, we have

$$
g(u)= \pm \int\left(\alpha u+\frac{a}{u}\right) \sqrt{\frac{u^{2}-h^{2}}{u^{2}-\left(\alpha u^{2}+a\right)^{2}}} d u .
$$

We now suppose that $M$ is spacelike, that is, $-u^{2}+h^{2}-u^{2} g^{\prime 2}>0$. Then, $M$ has constant mean curvature $\alpha$ if and only if $g=g(u)$ is a solution of the following differential equation

$$
-h^{2} u g^{\prime \prime}+u^{3} g^{\prime \prime}-2 h^{2} g^{\prime}+u^{2} g^{\prime}+u^{2} g^{\prime 3}=2 \alpha\left(-u^{2}+h^{2}-u^{2} g^{\prime 2}\right)^{\frac{3}{2}} .
$$

By the change of variables $-u^{2}+h^{2}=w^{2}$ and $\frac{u}{w} g^{\prime}=\cos y$, the equation (5.3) yields

$$
g(u)= \pm \int\left(\alpha u+\frac{a}{u}\right) \sqrt{\frac{-u^{2}+h^{2}}{u^{2}+\left(\alpha u^{2}+a\right)^{2}}} d u .
$$

Thus, we complete the proof.

Furthermore, if $a=\alpha=0$, then $g$ is constant. In this case, the parametrization of $M$ becomes

$$
x(u, v)=(u \sinh v, u \cosh v, b+h v), h \neq 0, b \in \mathbb{R} .
$$

It is nothing but a right helicoid of type $I$ in $\mathbb{E}_{1}^{3}$.

If $\alpha=0$ and $a \neq 0$, then we obtain

$$
g(u)= \begin{cases} \pm \int \frac{a}{u} \sqrt{\frac{u^{2}-h^{2}}{u^{2}-a^{2}}} d u & \text { if } M \text { is timelike } \\ \pm \int \frac{a}{u} \sqrt{\frac{h^{2}-u^{2}}{u^{2}+a^{2}}} d u & \text { if } M \text { is spacelike. }\end{cases}
$$

If $\alpha \neq 0$ and $a=0$, then we get

$$
g(u)= \begin{cases} \pm \int \alpha \sqrt{\frac{u^{2}-h^{2}}{1-\alpha^{2} u^{2}}} d u & \text { if } M \text { is timelike } \\ \pm \int \alpha \sqrt{\frac{h^{2}-u^{2}}{1+\alpha^{2} u^{2}}} d u & \text { if } M \text { is spacelike. }\end{cases}
$$

In this case, $g(u)$ cannot be expressed as a rational function unless $g$ is a constant. 
If $\alpha a \neq 0$, the function $g(u)$ cannot be expressed as a rational function. kind.

In such a case that $g(u)$ is a rational function, $M$ is said to be of rational

Thus, we conclude the following:

Corollary 5.4. A genuine helicoidal surface of rational kind with spacelike axis in a Minkowski 3-space $\mathbb{E}_{1}^{3}$ has pointwise 1-type Gauss map if and only if it is an open part of either a hyperbolic cylinder or a right helicoid of type I in $\mathbb{E}_{1}^{3}$.

Combining the results of the above theorem and a characterization of surfaces of revolution with pointwise 1-type Gauss map in a Minkowski 3-space ([11]), we have a characterization of helicoidal surfaces of rational kind with pointwise 1-type Gauss map in $\mathbb{E}_{1}^{3}$.

Corollary 5.5 (Characterization). A helicoidal surface of rational kind with spacelike axis has pointwise 1-type Gauss map in a Minkowski 3-space if and only if it is part of a hyperbolic cylinder, a hyperbolic cone or a right helicoid of type I in a Minkowski 3-space.

\section{Helicoidal surfaces with timelike axis in $\mathbb{E}_{1}^{3}$}

In this section, we examine the helicoidal surface with timelike axis in $\mathbb{E}_{1}^{3}$.

Let $M$ be a helicoidal surface with timelike axis parameterized by (4.9) for some smooth function $g$. If $M$ has pointwise 1-type Gauss map, then by the Lemma 4.1, the Laplacian of the Gauss map satisfies

$$
\Delta G=F(u)(G+(c, 0,0))
$$

for some constant $c$. If $M$ is timelike, we have the functions of $u, A_{2}, B_{2}$ and $D_{2}$ from (4.10) and (4.11)

$$
\begin{aligned}
& A_{2}=-h F\left(u^{2} g^{\prime 2}-u^{2}+h^{2}\right)^{3}, \\
& B_{2}=F u g^{\prime}\left(u^{2} g^{\prime 2}-u^{2}+h^{2}\right)^{3}, \\
& D_{2}=F\left(u^{2} g^{\prime 2}-u^{2}+h^{2}\right)^{3}\left(u-c \sqrt{u^{2} g^{\prime 2}-u^{2}+h^{2}}\right) .
\end{aligned}
$$

We now assume that $M$ is genuine, that is, $h \neq 0$. From the above equations, we get

$$
D_{2}=-\frac{A_{2}}{h}\left(u-c \sqrt{u^{2} g^{\prime 2}-u^{2}+h^{2}}\right) .
$$

Using a simple algebraic calculation, we have

$$
D_{2}=-\frac{A_{2}}{h} u \text {. }
$$

Combining (6.1) and (6.2), we have $c F\left(u^{2} g^{\prime 2}-u^{2}+h^{2}\right)^{7 / 2}=0$. Since the function $F$ is nonzero and $u^{2} g^{\prime 2}-u^{2}+h^{2} \neq 0, c=0$. Similarly, we can deal 
with the matters if $M$ is spacelike. This means that the constant vector $C$ is zero vector and thus we have:

Theorem 6.1. If a genuine helicoidal surface with timelike axis in a Minkowski 3-space $\mathbb{E}_{1}^{3}$ has pointwise 1-type Gauss map, then it is of the first kind.

We immediately obtain the following:

Corollary 6.2. Let $M$ be a genuine helicoidal surface with timelike axis in $\mathbb{E}_{1}^{3}$. Then, $M$ has pointwise 1-type Gauss map if and only if $M$ has constant mean curvature.

We also get the following theorem.

Theorem 6.3. Let $M$ be a genuine helicoidal surface with timelike axis in a Minkowski 3-space $\mathbb{E}_{1}^{3}$. Then, $M$ has pointwise 1-type Gauss map if and only if $M$ is an open part of either a circular cylinder parameterized by (4.8) or the surface parameterized by

$$
x(u, v)=(g(u)+h v, u \cos v, u \sin v), u>0, h \neq 0,
$$

where

$$
g(u)= \begin{cases} \pm \int\left(\alpha u+\frac{a}{u}\right) \sqrt{\frac{h^{2}-u^{2}}{u^{2}-\left(\alpha u^{2}+a\right)^{2}}} d u & \text { if } M \text { is timelike } \\ \pm \int\left(\alpha u+\frac{a}{u}\right) \sqrt{\frac{u^{2}-h^{2}}{u^{2}+\left(\alpha u^{2}+a\right)^{2}}} d u & \text { if } M \text { is spacelike }\end{cases}
$$

for some constant $a$ and constant mean curvature $\alpha$.

Proof. Suppose that a genuine helicoidal surface $M$ with timelike axis in $\mathbb{E}_{1}^{3}$ has pointwise 1-type Gauss map.

If the function $f$ in the parametrization (2.3) is constant, $M$ is an open part of a circular cylinder which is proved in Lemma 4.1 .

If $f$ is not constant, we assume $M$ is parameterized by (4.9).

First, we consider the case that $M$ is timelike, that is, $u^{2}-h^{2}-u^{2} g^{\prime 2}<0$. Similarly to proof of Theorem 5.3, $M$ has constant mean curvature $\alpha$ if and only if $g=g(u)$ is a solution of the following differential equation

$$
h^{2} u g^{\prime \prime}-u^{3} g^{\prime \prime}+2 h^{2} g^{\prime}-u^{2} g^{\prime}+u^{2} g^{\prime 3}=2 \alpha\left(-u^{2}+h^{2}+u^{2} g^{\prime 2}\right)^{\frac{3}{2}} .
$$

If $h^{2}-u^{2}>0$, we put $h^{2}-u^{2}=w^{2}$. If we put $\frac{u}{w} g^{\prime}=\tan y$, the equation (6.3) is reduced to

$$
y^{\prime}+\frac{1}{u} \tan y=2 \alpha \sec y
$$

It yields $\sin y=\alpha u+\frac{a}{u}$ for some constant $a$. Thus, (6.3) gives rise to

$$
g(u)= \pm \int\left(\alpha u+\frac{a}{u}\right) \sqrt{\frac{h^{2}-u^{2}}{u^{2}-\left(\alpha u^{2}+a\right)^{2}}} d u .
$$

If $h^{2}-u^{2}<0$, we put $h^{2}-u^{2}=-w^{2}$. By the change of variables such as $\frac{u}{w} g^{\prime}=\sec y$, the equation (6.3) becomes

$$
-u \csc y \cot y y^{\prime}+\csc y=2 \alpha u \text {. }
$$


The same result could be derived as the case above.

Consequently, if $M$ is timelike, $g(u)$ is obtained by

$$
g(u)= \pm \int\left(\alpha u+\frac{a}{u}\right) \sqrt{\frac{h^{2}-u^{2}}{u^{2}-\left(\alpha u^{2}+a\right)^{2}}} d u .
$$

Next, we consider that $M$ is spacelike, that is, $u^{2}-h^{2}-u^{2} g^{\prime 2}>0$. Like the previous case, $M$ has constant mean curvature $\alpha$ if and only if $g=g(u)$ is a solution of the following differential equation

$$
-h^{2} u g^{\prime \prime}+u^{3} g^{\prime \prime}-2 h^{2} g^{\prime}+u^{2} g^{\prime}-u^{2} g^{\prime 3}=2 \alpha\left(u^{2}-h^{2}-u^{2} g^{\prime 2}\right)^{\frac{3}{2}} .
$$

Since $u^{2}-h^{2}>0$, we put $u^{2}-h^{2}=w^{2}$. If we make an appropriate change of variables like $\frac{u}{w} g^{\prime}=\sin y,(6.4)$ becomes

$$
u \sec ^{2} y y^{\prime}+\tan y=2 \alpha u,
$$

from which,

$$
g(u)= \pm \int\left(\alpha u+\frac{a}{u}\right) \sqrt{\frac{u^{2}-h^{2}}{u^{2}+\left(\alpha u^{2}+a\right)^{2}}} d u
$$

for some constant $a$. Thus, it completes the proof.

We now examine the cases upon the constants $\alpha$ and $a$.

If $a=\alpha=0$, then $g$ is constant. In this case, the surface $M$ is part of a right helicoid of type $I I$.

If $\alpha=0$ and $a \neq 0, g(u)$ is given by

$$
g(u)= \begin{cases} \pm \int \frac{a}{u} \sqrt{\frac{u^{2}-h^{2}}{a^{2}-u^{2}}} d u & \text { if } M \text { is timelike } \\ \pm \int \frac{a}{u} \sqrt{\frac{u^{2}-h^{2}}{u^{2}+a^{2}}} d u & \text { if } M \text { is spacelike. }\end{cases}
$$

In this case, the function $g(u)$ cannot be a rational function. If $\alpha \neq 0$ and $a=0, g(u)$ is given by

$$
g(u)= \begin{cases} \pm \int \alpha \sqrt{\frac{u^{2}-h^{2}}{\alpha^{2} u^{2}-1}} d u & \text { if } M \text { is timelike } \\ \pm \int \alpha \sqrt{\frac{u^{2}-h^{2}}{1+\alpha^{2} u^{2}}} d u & \text { if } M \text { is spacelike. }\end{cases}
$$

The function $g(u)$ is rational only if $a=0$ and $h^{2} \alpha^{2}=1$. In this case, $g(u)=$ $\pm u+b$ for some constant $b$. Thus, the parametrization of $M$ turns out to be

$$
x(u, v)=( \pm u+b+h v, u \cos v, u \sin v), \quad h \neq 0, b \in \mathbb{R} .
$$

In this case, the surface is a helicoidal surface of elliptic type. Moreover, if $\alpha a \neq 0, g$ is expressed as some elliptic functions or hypergeometric functions.

Thus, we have the following:

Corollary 6.4. Let $M$ be a genuine helicoidal surface of rational kind with timelike axis in $\mathbb{E}_{1}^{3}$. Then, it has pointwise 1-type Gauss map if and only if it is an open part of either right helicoid of type II or helicoidal surface of elliptic type of the form (6.5). 
Together with Theorem 6.3 and the results in [11], we have:

Corollary 6.5 (Characterization). Let $M$ be a helicoidal surface of rational kind with timelike axis in $\mathbb{E}_{1}^{3}$. Then, $M$ has pointwise 1-type Gauss map if and only if it is an open part of a circular cylinder, a right cone, a right helicoid of type II or a helicoidal surface of elliptic type in $\mathbb{E}_{1}^{3}$.

\section{Helicoidal surfaces with null axis in $\mathbb{E}_{1}^{3}$}

In this section, we study the helicoidal surface with null axis in $\mathbb{E}_{1}^{3}$.

Let $M$ be a helicoidal surface with null axis parameterized by (4.12). Suppose that $M$ is a spacelike helicoidal surface of rational kind with pointwise 1-type Gauss map of the second kind. Then, the Gauss map $G$ satisfies the condition $\Delta G=F(u)(G+(c, c, 0))$ for a nonzero constant $c$ by Lemma 4.1. Let $(\Delta G)_{i}$ be the $i$-th component of $\Delta G$ for $i=1,2,3$. From equation (4.13), we have

$$
\begin{gathered}
(\Delta G)_{1}=F(u)\left(\frac{u k^{\prime}+u+u v^{2}-v h}{\sqrt{4 u^{2} k^{\prime}-h^{2}}}+c\right), \\
(\Delta G)_{2}=F(u)\left(\frac{u k^{\prime}-u+u v^{2}-v h}{\sqrt{4 u^{2} k^{\prime}-h^{2}}}+c\right), \\
(\Delta G)_{3}=F(u)\left(\frac{2 u v-h}{\sqrt{4 u^{2} k^{\prime}-h^{2}}}\right) .
\end{gathered}
$$

Therefore, from the above equations with (4.14), we easily obtain

$$
F(u)=-\frac{2 X(u)}{\left(4 u^{2} k^{\prime}-h^{2}\right)^{3}},
$$

where $X(u)$ is defined by (4.15). Putting this function $F(u)$ in (7.1) or (7.2), with the help of (4.14) (4.15) and (4.16), we obtain

$$
\begin{aligned}
& u \sqrt{4 u^{2} k^{\prime}-h^{2}}\left(8 h^{2} k^{\prime}+7 h^{2} k^{\prime \prime} u-8{k^{\prime}}^{2} u^{2}+h^{2} k^{\prime \prime \prime} u^{2}-4 k^{\prime} k^{\prime \prime} u^{3}+6{k^{\prime \prime}}^{2} u^{4}\right. \\
& \left.-4 k^{\prime} k^{\prime \prime \prime} u^{4}\right)+2 c\left(h^{4}+4 h^{2} k^{\prime} u^{2}+9 h^{2} k^{\prime \prime} u^{3}+h^{2} k^{\prime \prime \prime} u^{4}-4 k^{\prime} k^{\prime \prime} u^{5}+8 k^{\prime \prime 2} u^{6}\right. \\
& \left.-4 k^{\prime} k^{\prime \prime \prime} u^{6}\right)=0 .
\end{aligned}
$$

Since $k(u)$ is a rational function, $Q(u)=\sqrt{4 u^{2} k^{\prime}-h^{2}}$ is also a rational function because of (7.4). If we rearrange (7.4) with respect to $Q$, we have

(7.5) $2 u Q^{3} Q^{\prime 2}-u Q^{4} Q^{\prime \prime}-3 Q^{4} Q^{\prime}=-2 c\left(2 Q^{4}-5 u Q^{3} Q^{\prime}+3 u^{2} Q^{2} Q^{\prime 2}-u^{2} Q^{3} Q^{\prime \prime}\right)$.

From now on, we regard the rational function $Q$ as a complex meromorphic function. Let $Q(z)=\frac{q(z)}{p(z)}$, where $p$ and $q$ are relatively prime polynomials. 
First, we show that $q(z)=a z^{m}$ for some constant $a$ and a positive integer $m$. Suppose $q\left(z_{0}\right)=0$. It implies that $Q\left(z_{0}\right)=0$. Then

$$
Q(z)=\sum_{n=k}^{\infty} a_{n}\left(z-z_{0}\right)^{n}
$$

for some $k \geq 1$ and $a_{k} \neq 0$. If $z_{0} \neq 0, z=z_{0}+\left(z-z_{0}\right)$ and $z^{2}=z_{0}^{2}+2 z_{0}(z-$ $\left.z_{0}\right)+\left(z-z_{0}\right)^{2}$. If we compare the lowest degree of both sides of (7.5) after putting $z$ in (7.5) instead of $u$, we see that the lowest degree of the left hand side of $(7.5)$ is $5 k-2$ and that of the right hand side is $4 k-2$. Hence the coefficient of term of degree $4 k-2$ is zero, that is, $-2 c\left(2 k^{2}+k\right) z_{0}^{2} a_{k}{ }^{4}=0$, which is a contradiction. Thus, $q(z)=a z^{m}$ for some constant $a$ and a positive integer $m$ and so

$$
Q(z)=\frac{a z^{m}}{p(z)} .
$$

Let $p(z)=z^{k}+a_{1} z^{k-1}+a_{2} z^{k-2}+\cdots+a_{k}$. Since $p$ and $q$ are relatively prime polynomials, $a_{k} \neq 0$. The series expansion of $Q(z)$ at $z=0$ looks like $Q(z)=a z^{l}+a_{1} z^{l+1}+a_{2} z^{l+2}+\cdots, l \geq 1$. Then, the lowest degree of the right hand side of (7.5) is $5 l-1$ and that of the left hand side is $4 l$. If $l>1$, then the coefficient of term with degree $4 l$ must be zero, that is, $-4 c a^{4}(l-1)^{2}=0$, this is also a contradiction. If $l=1$, then the lowest degree of the left hand side of (7.5) is 5 and that of the right hand side is 4 . Hence, the coefficient of term with degree 4 must be zero, that is, $a=0$. Therefore, $Q(z)=0$, a contradiction. Thus, $m=0$ and $Q(z)=\frac{a}{p(z)}$. The polynomial $p(z)$ can be written as $p(z)=\left(z-\alpha_{1}\right)\left(z-\alpha_{2}\right) \cdots\left(z-\alpha_{k}\right)$ for some complex numbers $\alpha_{1}, \alpha_{2}, \ldots, \alpha_{k}\left(\alpha_{k} \geq 1\right)$. Since

$$
\frac{1}{z-\alpha_{1}}=\frac{1}{z}+\frac{\alpha_{1}}{z^{2}}+\frac{\alpha_{1}^{2}}{z^{3}}+\cdots\left(|z|>\left|\alpha_{1}\right|\right),
$$

the complex meromorphic function $Q(z)$ has the form

$$
Q(z)=\frac{a}{p(z)}=\frac{a}{z^{k}}+\frac{a_{1}}{z^{k+1}}+\frac{a_{2}}{z^{k+2}}+\cdots(|z|>r)
$$

for some $r>0$. Putting (7.6) into (7.5) and comparing the degrees of terms in the both sides, the lowest degree of terms in $1 / z$ 's of the left hand side is $4 k+2$ and that of the right hand side is $4 k$. Therefore, the coefficient in the term with degree $4 k$ in $1 / z$ must be zero. In other words, $-2 c a^{4}(k+1)^{2}(k+2)=0$. Hence, $a=0$ and so $Q(z)=0$. This is a contradiction. Consequently, if $c \neq 0$, (7.5) does not hold if $Q$ is a rational function. The case of timelike surface with null axis is similarly dealt with.

Thus, we have:

Theorem 7.1. Let $M$ be a helicoidal surface of rational kind with null axis in a Minkowski 3-space $\mathbb{E}_{1}^{3}$. Then, there exists no helicoidal surface of rational kind with pointwise 1-type Gauss map of the second kind. In other words, if the Gauss map is of pointwise 1-type, then it must be of the first kind. 
Next, we prove

Theorem 7.2. Let $M$ be a genuine helicoidal surface with null axis in $\mathbb{E}_{1}^{3}$. Then $M$ has pointwise 1-type Gauss map of the first kind if and only if it is an open part of a helicoidal surface of Enneper type, a helicoidal surface of hyperbolic type, a helicoidal surface of de Sitter type or a helicoidal surface of parabolic type.

Proof. Let $M$ be a genuine helicoidal surface with null axis in $\mathbb{E}_{1}^{3}$ parameterized by (4.12). Then, $M$ has pointwise 1-type Gauss map of the first kind if and only if $M$ has constant mean curvature $\alpha$.

First, consider $M$ is spacelike, that is, $4 u^{2} k^{\prime}-h^{2}>0$.

Since $M$ has constant mean curvature $\alpha$, we get

$$
k^{\prime \prime} u^{3}-2 k^{\prime} u^{2}+h^{2}+\alpha\left(4 u^{2} k^{\prime}-h^{2}\right)^{3 / 2}=0 .
$$

If $\alpha=0$, that is, $M$ is minimal, $k(u)$ is obtained by

$$
k(u)=a u^{3}-\frac{h^{2}}{4 u}+b
$$

for some constants $a>0$ and $b$. Therefore, the parametrization of $M$ can be reduced to

$$
x(u, v)=\left(\begin{array}{ccc}
1+\frac{v^{2}}{2} & -\frac{v^{2}}{2} & v \\
\frac{v^{2}}{2} & 1-\frac{v^{2}}{2} & v \\
v & -v & 1
\end{array}\right)\left(\begin{array}{c}
-u+a u^{3} \\
u+a u^{3} \\
0
\end{array}\right)+\left(\begin{array}{c}
-\frac{h^{2}}{4 u}+h v+b \\
-\frac{h^{2}}{4 u}+h v+b \\
0
\end{array}\right) .
$$

Thus, it is part of a spacelike helicoidal surface of Enneper type.

Suppose $\alpha \neq 0$. The equation (7.7) is a Bernoulli's differential equation and can be solved as

$$
k(u)=\frac{1}{4} \int\left(\frac{u^{2}}{\left(\alpha u^{2}+a\right)^{2}}+\frac{h^{2}}{u^{2}}\right) d u
$$

for some constant $a$. If $a=0$, then $k(u)=-\frac{1}{4 \alpha^{2} u}-\frac{h^{2}}{4 u}+b$ for some constant $b$. Thus, the parametrization of $M$ is reduced to

$$
x(u, v)=\left(\begin{array}{ccc}
1+\frac{v^{2}}{2} & -\frac{v^{2}}{2} & v \\
\frac{v^{2}}{2} & 1-\frac{v^{2}}{2} & v \\
v & -v & 1
\end{array}\right)\left(\begin{array}{c}
-u-\frac{1}{4 \alpha^{2} u} \\
u-\frac{1}{4 \alpha^{2} u} \\
0
\end{array}\right)+\left(\begin{array}{c}
-\frac{h^{2}}{4 u}+h v+b \\
-\frac{h^{2}}{4 u}+h v+b \\
0
\end{array}\right),
$$

which is part of a helicoidal surface of hyperbolic type.

If $\alpha a \neq 0$, then $k(u)$ is given by

$$
k(u)=-\frac{1}{4}\left(\frac{h^{2}}{u}+\frac{u}{2 \alpha\left(\alpha u^{2}+a\right)}-\frac{\tan ^{-1}\left(\sqrt{\frac{\alpha}{a}} u\right)}{2 \sqrt{|a|}|\alpha|^{3 / 2}}\right) \quad \text { if } \quad \alpha a>0
$$

or

$$
k(u)=-\frac{1}{4}\left(\frac{h^{2}}{u}+\frac{u}{2 \alpha\left(\alpha u^{2}-a\right)}+\frac{\tanh ^{-1}\left(\sqrt{\left|\frac{\alpha}{a}\right|} u\right)}{2 \sqrt{|a|}|\alpha|^{3 / 2}}\right) \quad \text { if } \quad \alpha a<0 .
$$


In this case, if $\alpha a>0$, the parametrization of $M$ can be written as

$$
\begin{aligned}
x(u, v)= & \left(\begin{array}{ccc}
1+\frac{v^{2}}{2} & -\frac{v^{2}}{2} & v \\
\frac{v^{2}}{2} & 1-\frac{v^{2}}{2} & v \\
v & -v & 1
\end{array}\right)\left(\begin{array}{c}
-u-\frac{u}{8 \alpha\left(\alpha u^{2}+a\right)}+\frac{\tan ^{-1}\left(\sqrt{\frac{\alpha}{a}} u\right)}{8 \sqrt{|a|}|\alpha|^{3 / 2}} \\
u-\frac{u}{8 \alpha\left(\alpha u^{2}+a\right)}+\frac{\tan ^{-1}\left(\sqrt{\frac{\alpha}{a}} u\right)}{8 \sqrt{|a|}|\alpha|^{3 / 2}} \\
0
\end{array}\right) \\
& +\left(\begin{array}{c}
-\frac{h^{2}}{4 u}+h v+b \\
-\frac{h^{2}}{4 u}+h v+b \\
0
\end{array}\right)
\end{aligned}
$$

or, if $\alpha a<0$,

$$
\begin{aligned}
x(u, v)= & \left(\begin{array}{ccc}
1+\frac{v^{2}}{2} & -\frac{v^{2}}{2} & v \\
\frac{v^{2}}{2} & 1-\frac{v^{2}}{2} & v \\
v & -v & 1
\end{array}\right)\left(\begin{array}{c}
-u-\frac{u}{8 \alpha\left(\alpha u^{2}-a\right)}-\frac{\tanh ^{-1}\left(\sqrt{\mid \frac{\alpha}{a}} u\right)}{8 \sqrt{|a|}|\alpha|^{3 / 2}} \\
u-\frac{u}{8 \alpha\left(\alpha u^{2}-a\right)}-\frac{\tanh ^{-1}\left(\sqrt{\left|\frac{\alpha}{a}\right|} u\right)}{8 \sqrt{|a|}|\alpha|^{3 / 2}} \\
0
\end{array}\right) \\
& +\left(\begin{array}{c}
-\frac{h^{2}}{4 u}+h v+b \\
-\frac{h^{2}}{4 u}+h v+b \\
0
\end{array}\right) .
\end{aligned}
$$

We call such a surface a helicoidal surface of parabolic type $I^{+}$or $I^{-}$according to the signature of $\alpha a$.

Similarly we can deal with the case that $M$ is timelike, that is, $4 u^{2} k^{\prime}-h^{2}<0$.

If $\alpha=0$, then $M$ is nothing but part of a timelike helicoidal surface of Enneper type. In this case, $k(u)$ is obtained by $k(u)=a u^{3}-\frac{h^{2}}{4 u}+b$ for some constants $a<0$ and $b$.

If $\alpha \neq 0$, then, $k(u)$ is given by

$$
k(u)=\frac{1}{4} \int\left(-\frac{u^{2}}{\left(\alpha u^{2}+a\right)^{2}}+\frac{h^{2}}{u^{2}}\right) d u
$$

for some constant $a$. If $a=0$, then $k(u)=\frac{1}{4 \alpha^{2} u}-\frac{h^{2}}{4 u}+b$ for some constant $b$. Hence, the parametrization of $M$ is reduced to

$$
x(u, v)=\left(\begin{array}{ccc}
1+\frac{v^{2}}{2} & -\frac{v^{2}}{2} & v \\
\frac{v^{2}}{2} & 1-\frac{v^{2}}{2} & v \\
v & -v & 1
\end{array}\right)\left(\begin{array}{c}
-u+\frac{1}{4 \alpha^{2} u} \\
u+\frac{1}{4 \alpha^{2} u} \\
0
\end{array}\right)+\left(\begin{array}{c}
-\frac{h^{2}}{4 u}+h v+b \\
-\frac{h^{2}}{4 u}+h v+b \\
0
\end{array}\right),
$$

which is part of a helicoidal surface of de Sitter type.

If $\alpha a \neq 0$, then $k(u)$ is given by

or

$$
k(u)=-\frac{1}{4}\left(\frac{h^{2}}{u}-\frac{u}{2 \alpha\left(\alpha u^{2}+a\right)}+\frac{\tan ^{-1}\left(\sqrt{\frac{\alpha}{a}} u\right)}{2 \sqrt{|a|}|\alpha|^{3 / 2}}\right) \quad \text { if } \quad \alpha a>0
$$

$$
k(u)=-\frac{1}{4}\left(\frac{h^{2}}{u}-\frac{u}{2 \alpha\left(\alpha u^{2}-a\right)}-\frac{\tanh ^{-1}\left(\sqrt{\left|\frac{\alpha}{a}\right|} u\right)}{2 \sqrt{|a|}|\alpha|^{3 / 2}}\right) \quad \text { if } \quad \alpha a<0 .
$$


Hence, if $\alpha a>0$, the parametrization of $M$ can be expressed as

$$
\begin{aligned}
x(u, v)= & \left(\begin{array}{ccc}
1+\frac{v^{2}}{2} & -\frac{v^{2}}{2} & v \\
\frac{v^{2}}{2} & 1-\frac{v^{2}}{2} & v \\
v & -v & 1
\end{array}\right)\left(\begin{array}{c}
-u+\frac{u}{8 \alpha\left(\alpha u^{2}+a\right)}-\frac{\tan ^{-1}\left(\sqrt{\frac{\alpha}{a}} u\right)}{8 \sqrt{|a|}|\alpha|^{3 / 2}} \\
u+\frac{u}{8 \alpha\left(\alpha u^{2}+a\right)}-\frac{\tan ^{-1}\left(\sqrt{\frac{\alpha}{a}} u\right)}{8 \sqrt{|a|}|\alpha|^{3 / 2}} \\
0
\end{array}\right) \\
& +\left(\begin{array}{c}
-\frac{h^{2}}{4 u}+h v+b \\
-\frac{h^{2}}{4 u}+h v+b \\
0
\end{array}\right)
\end{aligned}
$$

or, if $\alpha a<0$,

$$
\begin{aligned}
x(u, v)= & \left(\begin{array}{ccc}
1+\frac{v^{2}}{2} & -\frac{v^{2}}{2} & v \\
\frac{v^{2}}{2} & 1-\frac{v^{2}}{2} & v \\
v & -v & 1
\end{array}\right)\left(\begin{array}{c}
-u+\frac{u}{8 \alpha\left(\alpha u^{2}-a\right)}+\frac{\tanh ^{-1}\left(\sqrt{\left|\frac{\alpha}{a}\right|} u\right)}{8 \sqrt{|a|}|\alpha|^{3 / 2}} \\
u+\frac{u}{8 \alpha\left(\alpha u^{2}-a\right)}+\frac{\tanh ^{-1}\left(\sqrt{\left|\frac{\alpha}{a}\right|} u\right)}{8 \sqrt{|a|}|\alpha|^{3 / 2}} \\
0
\end{array}\right) \\
& +\left(\begin{array}{c}
-\frac{h^{2}}{4 u}+h v+b \\
-\frac{h^{2}}{4 u}+h v+b \\
0
\end{array}\right) .
\end{aligned}
$$

A timelike surface $M$ described above is called a helicoidal surface of parabolic type $I I^{+}$or $I I^{-}$according to the signature of $\alpha a$.

The converse is very straightforward. It completes the proof.

In particular, if $\alpha a \neq 0$, then $k(u)$ cannot be expressed as a rational function. Therefore, we have:

Corollary 7.3. Let $M$ be a genuine rational helicoidal surface with null axis in $\mathbb{E}_{1}^{3}$. Then $M$ has pointwise 1-type Gauss map if and only if it is an open part of a helicoidal surface of Enneper type, a helicoidal surface of hyperbolic type or a helicoidal surface of de Sitter type.

Putting together with the results described above and theorems in [11], we give a following characterization.

Corollary 7.4. (Characterization) A helicoidal surface of rational kind with null axis in a Minkowski 3-space has pointwise 1-type Gauss map if and only if it is part of Enneper's surface of second kind, a de-Sitter space, a hyperbolic space, a helicoidal surface of Enneper type, a helicoidal surface of hyperbolic type or a helicoidal surface of de Sitter type in $\mathbb{E}_{1}^{3}$.

\section{References}

[1] C. Baikoussis and D. E. Blair, On the Gauss map of ruled surfaces, Glasgow Math. J. 34 (1992), no. 3, 355-359.

[2] C. Baikoussis and L. Verstraelen, On the Gauss map of helicoidal surfaces, Rend. Sem. Mat. Messina Ser. II 2(16) (1993), 31-42. 
[3] C. C. Beneki and T. Koufogiorgos, Helicoidal surfaces with prescribed mean or Gaussian curvature, J. Geom. 63 (1998), no. 1-2, 25-29.

[4] C. C. Beneki, G. Kaimakamis, and B. J. Papantoniou, Helicoidal surfaces in threedimensional Minkowski space, J. Math. Anal. Appl. 275 (2002), no. 2, 586-614.

[5] B.-Y. Chen, Total Mean Curvature and Submanifolds of Finite Type, Series in Pure Mathematics, 1. World Scientific Publishing Co., Singapore, 1984.

[6] Finite Type Submanifolds and Generalizations, University of Rome, 1985.

[7] B.-Y. Chen, M. Choi, and Y. H. Kim, Surfaces of revolution with pointwise 1-type Gauss map, J. Korean Math. Soc. 42 (2005), no. 3, 447-455.

[8] B.-Y. Chen and P. Piccinni, Submanifolds with finite type Gauss map, Bull. Austral. Math. Soc. 35 (1987), no. 2, 161-186.

[9] M. Choi and Y. H. Kim, Characterization of the helicoid as ruled surfaces with pointwise 1-type Gauss map, Bull. Korean Math. Soc. 38 (2001), no. 4, 753-761.

[10] F. Ji and Z. H. Hou, Helicoidal surfaces under the cubic screw motion in Minkowski 3-space, J. Math. Anal. Appl. 318 (2006), no. 2, 634-647.

[11] U.-H. Ki, D.-S. Kim, Y. H. Kim, and Y.-M. Roh, Surfaces of revolution with pointwise 1-type Gauss map in Minkowski 3-space, Taiwanese J. Math. 13 (2009), no. 1, 317-338.

[12] Y. H. Kim and D. W. Yoon, Ruled surfaces with pointwise 1-type Gauss map, J. Geom. Phys. 34 (2000), no. 3-4, 191-205.

[13] - On the Gauss map of ruled surfaces in Minkowski space, Rocky Mountain J. Math. 35 (2005), no. 5, 1555-1581.

Miekyung Choi

Department of Mathematics

KYUNGPOOK NATIONAL UNIVERSITY

TAEGU 702-701, KoreA

E-mail address: mkchoi@knu.ac.kr

YOUNG Ho KIM

Department of Mathematics

KyUngPoOK National University

TAEGU 702-701, KoreA

E-mail address: yhkim@knu.ac.kr

Huili LiU

Department of Mathematics

Northeastern University

Shenyang 110004, P. R. ChinA

E-mail address: liuhl@mail.neu.edu.cn

DAE WON YOON

Department of Mathematics EDUCATION AND RINS

Gyeongsang National University

Jinju 660-701, KoreA

E-mail address: dwyoon@gsnu.ac.kr 